\title{
Contaminación por nitrógeno en el sistema "río-acuífero aluvial" de la cuenca del Jarama (Comunidad de Madrid, España) ¿Origen agrícola o urbano?
}

\author{
M. Arauzo ${ }^{* 1}$, J. J. Martínez-Bastida ${ }^{1,2}$ y M. Valladolid ${ }^{3}$ \\ ${ }^{1}$ Dpto. de Contaminación Ambiental, Centro de Ciencias Medioambientales-CSIC, Serrano 115 dpdo. 28006 \\ Madrid, España. \\ 2 martinez.bastida@ccma.csic.es \\ 3 Dpto. de Biodiversidad y Biología Evolutiva, Museo Nacional de Ciencias Naturales-CSIC, José Gutiérrez \\ Abascal 2, 28006 Madrid, España. E-mail: marval@ mncn.csic.es
}

* Autor responsable de la correspondencia: mercedes@ccma.csic.es

Recibido: 22/9/07

Aceptado: 11/4/08

\begin{abstract}
Nitrogen pollution in the "river-alluvial aquifer" system of the Jarama catchment (Comunidad de Madrid, Spain): Agricultural or urban origin?

A hydrochemical characterization of the "river-alluvial aquifer" system in the Quaternary deposits of the Jarama catchment has been performed, including the rivers Jarama, Henares, Manzanares, Tajuña and a part of Tajo, as well as the alluvial aquifer associated to the said fluvial net. The roles of agriculture and urban uses have been explored as possible sources of nitrogen pollution in surface and underground water resources.

Two sampling campaigns were performed, in March (at the end of the winter) and in August of 2005 (when irrigation water demand was at its highest), with water samples collected at 35 sampling stations (16 wells on the alluvial aquifer and 19 fluvial stations). The medium and low areas of the Manzanares, Jarama, Tajuña, and Tajo rivers did not meet the standards of quality established by the Tajo Catchment Hydrologic, due to high levels of ammonia, QOD and. electric conductivity, attributable to the incorporation of water treatment effluents originating from urban areas, (in some areas, conductivity levels could be explained by the geological context). The medium and low areas of the alluvial aquifer (in each subcatchment) showed very high values of nitrate concentration and conductivity, making its use unsuitable for drinking and restricting it for agricultural irrigation. A clear concordance was found between the spatial distribution of nitrate pollution in the alluvial aquifer and the agricultural irrigated areas. Using the N/P ratio as an indicator of the agricultural or urban origin it is interpreted that nitrogen pollution is mainly of urban origin in the rivers and of agricultural precedence in the alluvial aquifer (attributable to the bad management of fertilization and irrigation). The problem is compounded when water from nitrogen polluted rivers is used for irrigation, transferring nitrogen of fluvial origin into the alluvial aquifer with the irrigation return flows (urban nitrogen + fertilizers nitrogen). On the other hand, it has been observed that the excess of irrigation reverts the natural dynamic of the aquifer, producing rises of the phreatic level in extensive areas during the summer. These results provide a scientific basis to consider the declaration of the Quaternary alluvial deposits of the Jarama catchment as a Vulnerable Zone to nitrate contamination of agricultural origin, as established in the Directive 91/676/EEC.
\end{abstract}

Key words: "River-alluvial aquifer", Jarama catchment, nitrogen, nitrate, ammonia, irrigated agriculture, urban effluents, Vulnerable Zones to nitrate pollution.

\section{RESUMEN}

Contaminación por nitrógeno en el sistema "río-acuífero aluvial" de la cuenca del Jarama (Comunidad de Madrid, España) ¿Origen agrícola o urbano?

Se ha realizado una caracterización hidroquímica del sistema "río-acuífero aluvial" situado en los depósitos cuaternarios de la cuenca del Jarama, incluyendo los ríos Jarama, Henares, Manzanares, Tajuña y una parte del Tajo, así como el acuífero aluvial asociado a dicha red fluvial. Se ha explorado el papel de los usos agrícolas y urbanos como fuentes potenciales de contaminación por nitrógeno de los recursos hídricos superficiales y subterráneos. 
Se realizaron dos campañas de muestreo, en marzo (final del invierno) y agosto de 2005 (cuando la demanda de agua para riego era máxima), con recogida de muestras de agua en 35 estaciones de muestreo (16 pozos sobre el acuífero aluvial y 19 estaciones fluviales). En los tramos medios y bajos de los ríos Manzanares, Jarama, Tajuña y Tajo no se alcanzaban los objetivos de calidad establecidos en el Plan Hidrológico de la Cuenca del Tajo, debido a los elevados valores de amonio, DQO y conductividad eléctrica, atribuibles a la incorporación de efluentes de depuradora procedentes de las áreas urbanas (los niveles de conductividad pueden explicarse por el propio contexto geológico en algunas zonas). Los tramos medios y bajos del acuífero aluvial (en cada subcuenca) mostraron valores muy elevados de nitrato y conductividad, imposibilitando su uso para el abastecimiento y restringiéndolo para el riego agrícola. Se aprecia una clara concordancia entre la distribución espacial de la contaminación por nitrato en el acuífero aluvial y las zonas agrícolas de regadío. Utilizando el cociente N/P como indicador del origen agrícola o urbano de la contaminación por nitrógeno, se interpreta una procedencia principalmente urbana en los ríos y una procedencia agrícola en el acuífero aluvial (atribuible a las malas prácticas de riego y abonado). El problema se complica cuando los riegos se realizan con aguas fluviales contaminadas por nitrógeno, produciéndose un trasvase de nitrógeno de origen fluvial hacia el acuífero aluvial con los retornos de riego (nitrógeno urbano + nitrógeno de los fertilizantes). Por otra parte, se ha observado que el riego en exceso invierte la dinámica hídrica natural del acuífero, produciendo ascensos en el nivel freático en amplias zonas durante el verano. Estos resultados proporcionan una base científica para considerar la de claración de los aluviales cuaternarios de la cuenca del Jarama como Zona Vulnerable a la contaminación por nitrato de origen agrícola, tal como se establece en la Directiva 91/676/CEE.

Palabras clave: “Río-acuífero aluvial”, cuenca del Jarama, nitrógeno, nitrato, amonio, agricultura de regadío, efluentes urbanos, Zonas Vulnerables a la contaminación por nitratos.

\section{INTRODUCCIÓN}

La contaminación difusa tiende a adquirir cada vez mayor protagonismo en la degradación de los recursos hídricos (Knapp, 2005), si bien, en territorios intensamente antropizados, con frecuencia no es fácil identificar la procedencia de los contaminantes en las masas de agua. Aspectos generalmente ignorados, como el estudio de las interacciones entre el río y su acuífero aluvial asociado, o el papel de los usos del territorio en el deterioro de la calidad del agua, pueden proporcionar una información esencial para la gestión sostenible de los recursos hídricos a escala de cuenca.

Bajo las premisas de observación a escala de cuenca e interpretación integral del sistema "ríoacuífero aluvial", se ha realizado una caracterización hidroquímica del sistema "río-acuífero aluvial" situado en los depósitos cuaternarios de la cuenca del río Jarama (Comunidad de Madrid, España), incluyendo el río Jarama, sus afluentes Henares, Manzanares y Tajuña, una parte del río Tajo (a su paso por la Comunidad de Madrid), y el acuífero aluvial asociado a dicha red fluvial. Los objetivos principales de la investigación fueron determinar los niveles de contaminación por nitrógeno en los ríos y en el acuífero aluvial, identificar las formas químicas de nitrógeno dominantes en cada parte del sistema río-acuífero y explorar el papel de las principales fuentes de nitrógeno en el proceso de contaminación (lixiviación de fertilizantes en las zonas agrícolas de regadío y vertido de efluentes de depuradora procedentes de las áreas urbanas).

La cuenca del río Jarama es la más extensa y antropizada de la Comunidad de Madrid. Sus recursos hídricos superficiales se ven sometidos a una intensa e insostenible demanda de agua y a una elevada carga contaminación del origen urbano e industrial. Existe abundante información sobre el deficiente estado de calidad en los tramos medios y bajos del río Jarama y algunos de sus tributarios (Confederación Hidrográfica del Tajo, 2005 ${ }^{a}$ ), siendo el nitrógeno uno de los contaminantes de mayor presencia. De hecho, en el Plan Hidrológico de la Cuenca del Tajo (R.D. 1664/1998; Orden 18236 de 13 de agosto de 1999) se reconoce implícitamente la imposibilidad de recuperación de la calidad del agua en esos tramos, al excluir entre sus objetivos de ca- 
lidad los usos para abastecimiento, vida piscícola y baño. Por otra parte, sobre los depósitos aluviales de la cuenca del Jarama se sitúan las denominadas Vegas de la Comunidad de Madrid (Vega del Jarama, del Henares, del Tajuña y del Tajo), cuyas economías se basan principalmente en la agricultura de regadío. La infiltración en el terreno de aguas con alto contenido en nitrato, como resultado de una excesiva fertilización nitrogenada y unas prácticas de riego poco optimizadas (Hall et al., 2001), contribuye al deterioro de los recursos hídricos subterráneos (Nixon et al., 2000; Ball et al., 2005; Heathwaite et al., 2005; Abrantes et al., 2006). El Ministerio de Medio Ambiente (2001) señala que la cuenca media-baja del río Jarama es una de las que soportan mayores aportes globales de nitrógeno en España, debido a los usos agrícolas.

El exceso de nitrato en las aguas destinadas a abastecimiento puede afectar a la salud humana (Varela, 1994; Morales-Suárez et al., 1995; Sandor et al., 2001; Forman, 2004; Thorpe \& Shirmohammadi, 2005) y contribuye al desarrollo de procesos de eutrofización en las aguas superficiales (FAO/CEPE, 1991; Neal \& Jarvie, 2005). La Directiva 91/676/CEE regula la protección de las aguas contra la contaminación por nitrato de origen agrícola. En esta Directiva se define como Zona Vulnerable a aquella superficie del territorio cuya escorrentía o filtración afecte o pueda afectar a la contaminación del agua por el nitrato procedente de los fertilizantes, considerándose aguas afectadas por la contaminación aquellas con contenido en nitrato superior a $50 \mathrm{mg} / \mathrm{l}$, así como las que manifiestan una tendencia que haga prever la necesidad de medidas de protección. La Directiva Marco del Agua fija los plazos de cumplimiento de los objetivos medioambientales en las Zonas Vulnerables y determina los programas de seguimiento de las masas de agua. Asimismo, adopta como unidad de planificación para el control de los procesos de contaminación puntual y difusa la cuenca fluvial.

Las prospecciones de masas de agua contaminadas por nitrato en las que se ha incluido el territorio de la Comunidad de Madrid se han circunscrito a los acuíferos del Terciario, no habiendo dado lugar declaración de Zo- nas Vulnerables (Instituto Tecnológico Geominero de España, 1998; Ministerio de Medio Ambiente, 2001; Confederación Hidrográfica del Tajo, $2005^{b}$ ). La naturaleza permeable del sustrato y la escasa profundidad del nivel freático en los terrenos aluviales contribuyen a incrementar la vulnerabilidad de las masas de agua subyacentes. Sin embargo, a pesar de la combinación de factores de riesgo que convierten a los acuíferos aluviales de los depósitos cuaternarios de la Comunidad de Madrid en altamente vulnerables, hasta ahora no se había abordado su estudio. Navas et al. (1998) describen a la unidad cuaternaria como "de vulnerabilidad muy alta, constituida por depósitos fluviales de gravas y arenas, y en menor proporción limos y arcillas, con una zona no saturada altamente permeable y nivel freático a menos de $5 \mathrm{~m}$ de profundidad", si bien no detallan los procesos de contaminación que afectan a los recursos hídricos de los depósitos cuaternarios. La carencia de información sobre el estado de calidad del acuífero aluvial de la cuenca del Jarama podría explicarse por la gran dificultad en la localización de puntos de muestreo, puesto que existen ya pocos pozos accesibles (Las Vegas de la Comunidad de Madrid cuentan con sistemas de acequias para el riego, con agua procedente de la red fluvial, habiendo desaparecido la mayor parte de los pozos en el área aluvial). De ahí que uno de los objetivos parciales de este trabajo haya sido establecer una primera red de muestreo para la caracterización hidroquímica del acuífero aluvial de la cuenca del Jarama.

Los elevados niveles de nitrógeno en el sistema "río-acuífero aluvial" de la cuenca de Jarama precisa del esclarecimiento de las posibles fuentes de contaminación y de sus efectos sobre cada parte del sistema. Sin embargo, en áreas tan antropizadas como la que nos ocupa existe un importante grado de incertidumbre en el diagnóstico de las fuentes de contaminación que plantea diversos de interrogantes: ¿Dónde y cuándo se produce la contaminación por nitrógeno? ¿En que formas químicas? ¿Cómo afecta a cada parte del sistema "río-acuífero aluvial"? ¿Es atribuible a un origen urbano, agrícola o mixto? ¿Dónde ha de establecerse el límite de las responsabilidades? 


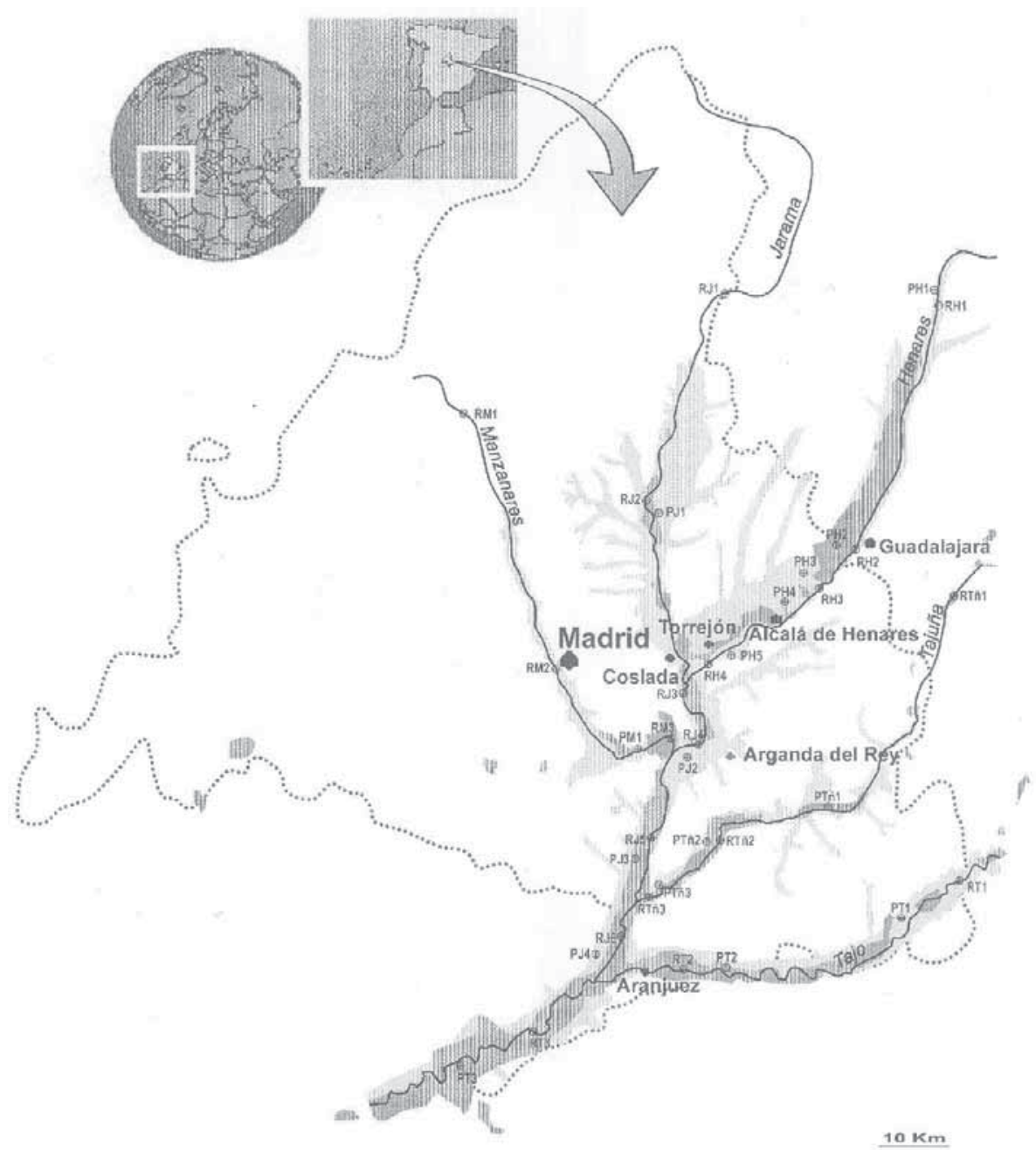

Figura 1. Localización de las estaciones de muestreo en los ríos y en el acuífero aluvial. En color gris claro: Extensión del acuífero aluvial sobre los depósitos cuaternarios de la cuenca del Jarama (incluyendo una parte de la cuenca del Tajo). En color gris oscuro: Distribución del área agrícola de regadío en la Comunidad de Madrid (fuente: MAPYA, 2005). Se muestra la situación de los principales núcleos urbanos (> 25000 habitantes). Location of the sampling stations on the rivers and alluvial aquifer. Light grey colour: Area of the alluvial aquifer over the quaternary deposits of the Jarama catchment (including a part of the Tajo catchment). Dark grey colour: Distribution of the irrigated agriculture area in the Comunidad de Madrid (source: MAPYA, 2005). Main urban areas are shown (> 25000 inhabitants).

\section{MATERIAL Y MÉTODOS}

\section{Área de estudio}

El área de estudio queda delimitada por la superficie que ocupan los depósitos aluviales del Cuaternario de la Comunidad de Madrid (España) sobre los que se sitúa el sistema "río-acuífero aluvial" de la cuenca del río Jarama, incluyendo el río Jarama y sus afluentes Henares, Manzanares y Tajuña, una parte del río Tajo (a su paso por la Comunidad de Madrid) y el acuífero aluvial asociado a dicha red fluvial.

El acuífero aluvial es de tipo libre y su extensión queda definida por la propia superficie aluvial $\left(1480 \mathrm{~km}^{2}\right)$. Su sustrato geológico lo constituyen gravas poligénicas, arenas y limos (primeras terrazas aluviales) y arenas, limos arenosos y cantos (fondos de valle), todos ellos depósitos de alta permeabilidad (ITGME-CAM, 1988). Los 
perfiles edáficos dominantes son de tipo Fluvisol y Luvisol, también muy permeables (Guerra y Monturiol, 1970; Monturiol y Alcalá 1990). Debido a su probable desconexión con el acuífero aluvial principal, las masas de agua de menor entidad situadas en las terrazas medias y altas no han sido incluidas en el estudio.

En la figura 1 se muestra un mapa de la red fluvial y se delimita la extensión de los depósitos cuaternarios, en los que se encuentra el acuífero aluvial. Las estaciones de muestreo de la red fluvial se denominaron $\mathrm{RJn}^{o}, \mathrm{RHn}^{o}, \mathrm{RMn}^{o}, \mathrm{Rtñn}^{o}$ y $\mathrm{RTn}^{o}$, mientras que los pozos para el muestro del acuífero aluvial se nombraron como $\mathrm{PJn}^{\circ}$, $\mathrm{PHn}^{o}, \mathrm{PMn}^{o}, \mathrm{Ptñn}^{o}$ y $\mathrm{PTn}^{o}$, asignándose una numeración creciente desde las zonas de cabecera hasta los tramos bajos y utilizando los siguientes códigos de cuenca: Jarama (J), Henares $(\mathrm{H})$, Manzanares (M), Tajuña (tñ) y Tajo (T). También se representan las superficies destinadas a agricultura de regadío en la Comunidad de Madrid (MAPYA, 2005), denominadas localmente Vegas del Jarama, el Henares, el Tajuña y el Tajo, y se sitúan los principales núcleos urbanos. Cabe destacar que sólo el área metropolitana de Madrid cuenta con una población de más de 5800000 habitantes, sobrepasándose en Alcalá de Henares, Torrejón, Coslada, Guadalajara y Aranjuez los 40000 habitantes. Según la localización de los principales núcleos urbanos (Fig. 1), los ríos que potencialmente podrían verse más afectados por el vertido de efluentes de depuradora serían el Manzanares, el Jarama, el Henares y el Tajo (aguas abajo de su confluencia con el Jarama).

En la Tabla 1 se muestran algunos parámetros de localización de las estaciones de muestreo y se especifican los objetivos de calidad establecidos en el Plan Hidrológico de la Cuenca del Tajo para cada tramo de los ríos y del acuífero aluvial.

\section{Metodología}

Del 6 al 8 de marzo y del 1 al 3 de agosto de 2005 se realizaron dos campañas de muestreo, con recogida de muestras de agua en 19 estaciones fluviales (ríos Jarama, Henares, Manzanares, Tajuña y Tajo) y en 16 pozos situados en el acuífero aluvial (Fig. 1). La elección de los periodos de muestreo responde a la necesidad de explorar los efectos de la agricultura de regadío sobre la calidad del agua y la dinámica hídrica en el sistema río-acuífero: periodo final del invierno (tras la recarga invernal, momento en el se esperaba que la superficie freática se encontrarse en su nivel más alto) y periodo de verano (después de la fertilización y durante la máxima demanda de agua para riego, con extracciones por bombeo desde los cauces fluviales).

En cada campaña se realizaron medidas in si$t u$ de temperatura del agua, porcentaje de saturación de oxígeno, $\mathrm{pH}$ y conductividad eléctrica en todas las estaciones de muestreo (ríos y acuífero). Para ello se utilizó un sistema multiparamétrico portátil conectado a multisonda y kit pH/redox modelo YSI 556. Las medidas de coordenadas UTM y altitud se realizaron mediante un GPS modelo Garmin GPS 12. Se efectuaron anotaciones sobre los usos del suelo en cada punto de muestreo (zona natural, uso terciario, erial, agricultura de regadío, tipo de cultivo, tipo de riego, etc.). En los pozos también se midió la profundidad del nivel freático, mediante un Hidronivel Meyer. Se extrajeron muestras de agua de tipo simple en los ríos, e integradas cada dos metros de profundidad en los pozos. Las muestras de agua de pozo se recogieron mediante una botella muestreadora de apertura horizontal de 2.51 , o bien usando un Hidronivel Meyer dotado de botella tomamuestras. Sobre cada muestra se analizaron los siguientes parámetros: Nitrógeno total, nitrato, nitrito, amonio, fósforo total, sulfatos, carbonatos, bicarbonatos, cloruros, calcio, potasio, magnesio, sodio, sílice y demanda química de oxígeno (DQO). Los aniones se determinaron mediante cromatografía iónica, mientras que para el análisis de cationes se utilizó la espectrometría de emisión ICP-AES (plasma de acoplamiento inductivo). Se realizaron clasificaciones del agua según la dureza y según el contenido iónico (diagrama de Piper). La DQO se determinó mediante la técnica del dicromato potásico (APHA, 1998). El nitrógeno total y el fósforo total por colorimetría, el primero mediante el método 2.6-dimetil fenol (Lange, 1998) y el segundo mediante el método del fosfomolibdeno 
Tabla 1. Parámetros de localización de las estaciones de muestreo, objetivos de calidad establecidos en el Plan Hidrológico de la Cuenca del Tajo y clasificaciones del agua según la dureza y el diagrama de Piper. Location parameters of the sampling stations, quality objectives established in the Tajo Catchment Hydrologic Plan and water classifications based on the water hardness and the Piper diagram.

\begin{tabular}{|c|c|c|c|c|c|c|c|}
\hline \multirow[t]{2}{*}{ Estación } & \multirow[t]{2}{*}{ Enclave } & \multirow[t]{2}{*}{ Coordenadas UTM } & \multirow{2}{*}{$\begin{array}{l}\text { Altitud } \\
\text { (m) }\end{array}$} & \multirow{2}{*}{$\begin{array}{c}\text { Objetivos de calidad } \\
\text { Plan Hidrológico }\end{array}$} & \multicolumn{2}{|r|}{ Dureza } & \multirow{2}{*}{$\begin{array}{c}\text { Diagrama de Piper } \\
\text { Clasificación }\end{array}$} \\
\hline & & & & & ${ }^{\circ} \mathbf{F}$ & Clasificación & \\
\hline RH1 & Río Henares & 30T 04902574521152 & 700 & A3 & 50 & dura & sulfatada cálcica \\
\hline $\mathrm{RH} 2$ & Río Henares & 30T 04804894491619 & 685 & A3 & 60 & muy dura & sulfatada cálcica \\
\hline RH3 & Río Henares & 30T 04804584491621 & 622 & - & 49 & dura & sulfatada cálcica \\
\hline RH4 & Río Henares & 30T 04611004476681 & 566 & - & 48 & dura & sulfatada cálcica \\
\hline RM1 & Río Manzanares & 30T 04249984510670 & 910 & A2 & 1 & muy blanda & bicarbonatada sódica \\
\hline RM2 & Río Manzanares & 30T 04367214479229 & 664 & - & 10 & blanda & bicarbonatada sódica \\
\hline RM3 & Río Manzanares & 30T 04554314463738 & 540 & - & 36 & dura & bicarbonatada sódica \\
\hline Rtñ 1 & Río Tajuña & 30T 04994744491742 & 755 & A2 & 31 & medianam. dura & bicarbonatada cálcica \\
\hline $\operatorname{Rt} \tilde{n} 2$ & Río Tajuña & 30T 04515114432779 & 511 & A3 & 74 & muy dura & sulfatada cálcica \\
\hline Rtñ3 & Río Tajuña & 30T 04512114442648 & 510 & A3 & 78 & muy dura & sulfatada cálcica \\
\hline RJ1 & Río Jarama & $30 \mathrm{~T} 04607294524400$ & 916 & $\mathrm{~A} 2$ & 22 & medianam. dulce & bicarbonatada cálcica \\
\hline $\mathrm{RJ} 2$ & Río Jarama & 30T 04518664492768 & 603 & $\mathrm{~A} 2$ & 37 & dura & sulfatada cálcica \\
\hline RJ3 & Río Jarama & 30T 04568644471829 & 551 & - & 36 & dura & sulfatada cálcica \\
\hline RJ4 & Río Jarama & 30T 04588424464220 & 533 & - & 49 & dura & sulfatada cálcica \\
\hline RJ5 & Río Jarama & 30T 04540764454079 & 520 & - & 39 & dura & sulfatada cálcica \\
\hline RJ6 & Río Jarama & 30T 04481534437763 & 507 & - & 45 & dura & sulfatada cálcica \\
\hline RT1 & Río Tajo & 30T 04994424450505 & 730 & A2 & 57 & muy dura & sulfatada cálcica \\
\hline RT2 & Río Tajo & 30T 04515074432708 & 514 & A2 & 93 & muy dura & sulfatada cálcica \\
\hline RT3 & Río Tajo & 30S 04369334423813 & 491 & $\mathrm{~A} 2$ & 59 & muy dura & sulf. clor. cálcica-sódica \\
\hline PH1 & Acuífero aluvial, Henares & 30T 04898864520641 & 706 & A3 & 30 & medianam. dura & sulfatada cálcica \\
\hline PH2 & Acuífero aluvial, Henares & 30T 04832814498618 & 652 & A3 & 75 & muy dura & sulfatada cálcica \\
\hline PH3 & Acuífero aluvial, Henares & 30T 04785994493268 & 634 & - & 58 & muy dura & sulf. bic. cál. magnésica \\
\hline PH4 & Acuífero aluvial, Henares & 30T 04757364485694 & 599 & - & 91 & muy dura & sulf. cálcico magnésica \\
\hline PH5 & Acuífero aluvial, Henares & 30T 04619974476072 & 576 & - & 168 & muy dura & sulf. cálcico-magnésica \\
\hline PM1 & Acuífero aluvial, Manzanares & 30T 04538864464873 & 546 & - & 178 & muy dura & sulfatada cálcica \\
\hline Ptñ1 & Acuífero aluvial, Tajuña & $30 \mathrm{~T} 04726444453911$ & 578 & A3 & 131 & muy dura & sulfatada cálcica \\
\hline Ptñ2 & Acuífero aluvial, Tajuña & 30T 04595274447349 & 540 & A3 & 104 & muy dura & sulfatada cálcica \\
\hline Ptñ3 & Acuífero aluvial, Tajuña & 30T 04524024443247 & 506 & A3 & 108 & muy dura & sulfatada cálcica \\
\hline PJ1 & Acuífero aluvial, Jarama & 30T 04528144491800 & 587 & $\mathrm{~A} 2$ & 56 & muy dura & sulfatada cálcica \\
\hline $\mathrm{PJ} 2$ & Acuífero aluvial, Jarama & $30 \mathrm{~T} 04589224463226$ & 537 & A2 & 54 & muy dura & sulfatada cálcica \\
\hline PJ3 & Acuífero aluvial, Jarama & 30T 04517764451772 & 510 & - & 67 & muy dura & sulfatada cálcica \\
\hline PJ4 & Acuífero aluvial, Jarama & 30T 04471484436826 & 487 & - & 203 & muy dura & clor. sulfatada sódica \\
\hline PT1 & Acuífero aluvial, Tajo & 30T 04840784438055 & 548 & A2 & 163 & muy dura & sulfatada cálcica \\
\hline PT2 & Acuífero aluvial, Tajo & 30T 04539974434530 & 501 & A2 & 115 & muy dura & sulfatada cálcica \\
\hline PT3 & Acuífero aluvial, Tajo & 30S 04293974420288 & 475 & A2 & 86 & muy dura & sulfatada cálcica \\
\hline
\end{tabular}

(Murphy \& Riley, 1962). Para la determinación del amonio se usó el método colorimétrico del indofenol azul (Lange, 1998).

El cociente N/P se calculó a partir de las medidas de nitrógeno total y de fósforo total, y se utilizó como un indicador del origen agrícola o urbano de la contaminación por nitrógeno: En aguas naturales no contaminadas, o cuando se sospecha que la procedencia del enriquecimiento en nitrógeno es de origen urbano (efluentes de depuradora) los valores de cociente N/P se encuentran en torno a 12-16 (San Diego-McGlonge et al., 2000). Valores más elevados (que pueden superar en uno y en dos órdenes de magnitud los anteriores) podrían indicar contaminación de origen agrícola. Esto se debe a que en suelos básicos o muy ricos en calcio, como los del área de estudio (Guerra y Monturiol, 1970; Monturiol y Alcalá 1990), o bien cuando el agua de riego es calcárea (Tabla 1), el fósforo pasa rápida- 
mente a ser insoluble, a diferencia del nitrato, y no presenta problemas de lixiviación (College of Agricultural Sciences, Agricultural Research and Cooperative Extension, 2001).

La variable denominada "superficie de regadío aguas arriba de cada pozo" se elaboró superponiendo el mapa de las superficies destinadas a agricultura de regadío (MAPYA, 2005) y los puntos de muestreo situados sobre el acuífero aluvial (Fig. 1), y estimando la distancia lineal máxima (en km) indicadora de la extensión agrícola aguas arriba de cada pozo, a fin explorar la relación entre la superficie dedicada al regadío en el área de influencia de cada punto de muestreo del acuífero y los niveles de contaminación por nitrato en el mismo.

Los mapas de isolíneas de concentración de nitrato en el acuífero aluvial se elaboraron a partir de las concentraciones de nitrato obtenidas en cada punto, trazando isolíneas por interpolación triangular e interpretativa, teniendo en cuenta el sentido del flujo del agua. En los mapas de isolíneas de nitrato también representa la distribución del contenido en nitrógeno total en los ríos de la cuenca.

Los datos de precitación se obtuvieron de una estación meteorológica modelo Vantage Pro Plus, situada en la zona media del área de estudio (Fin- ca Experimental La Poveda CCMA-CSIC). La información geológica y edáfica se recopiló a partir de ITGME-CAM (1988), Guerra y Monturiol (1970) y Monturiol y Alcalá (1990).

\section{RESULTADOS Y DISCUSIÓN}

La distribución del territorio destinado a la agricultura de regadío en la Comunidad de Madrid (MAPYA, 2005) presenta un apreciable solapamiento con los tramos medios y bajos del acuífero aluvial situado en los depósitos cuaternarios (Fig. 1). Durante la campaña de marzo se observó la dominancia de rastrojeras de cereal de invierno, mientras que en el verano el cultivo principal era el maíz, seguido de hortícolas y otras forrajeras. Cultivos arbóreos, viñedos, zonas de recuperación de soto, eriales y áreas destinadas a uso terciario, coexistían con los cultivos herbáceos, aunque en menor extensión. Durante la campaña de agosto se constató el uso generalizado del riego por inundación en los cultivos de maíz, alfalfa y arbóreos de las zonas correspondientes a las estaciones PH2, PH3, PJ3, PT1 y PT2, mientras que en el resto de la superficie dedicada al regadío se aplicaba riego por aspersión.

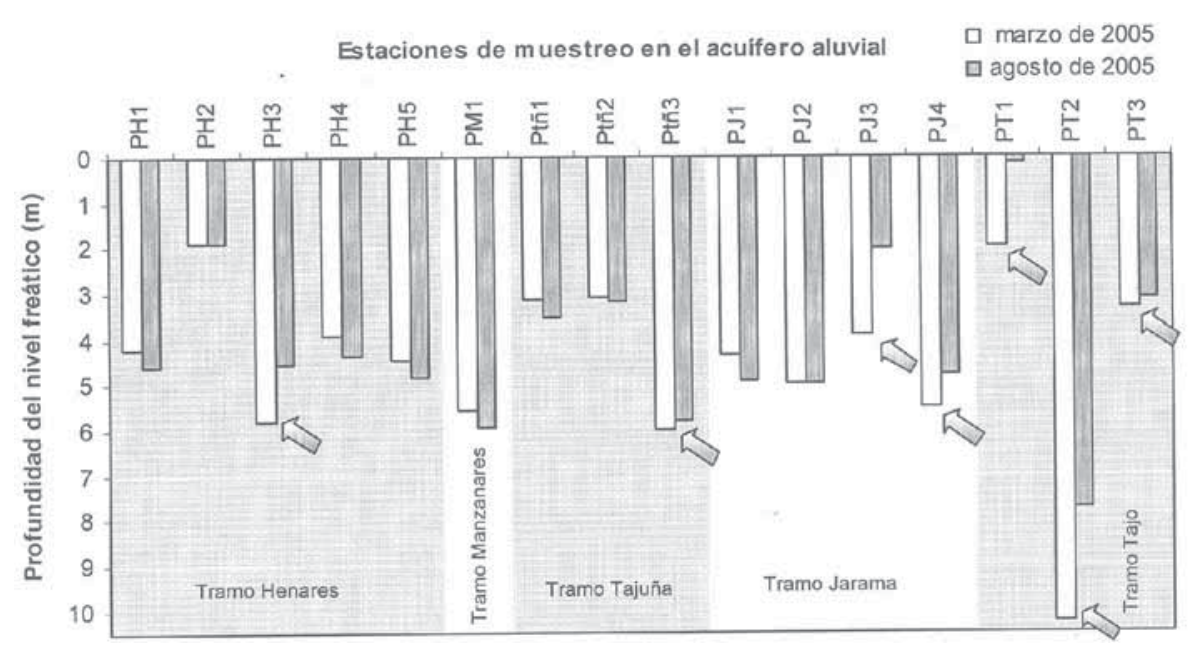

Figura 2. Nivel freático del acuífero aluvial al final del invierno (tras la recarga invernal) y durante el verano de 2005 (periodo de máxima demanda de agua para riego agrícola). Las flechas señalan los puntos del acuífero en los que se produjeron ascensos del nivel freático durante el verano. Phreatic level of the alluvial aquifer at the end of winter (after winter recharge) and during the summer of 2005 (period of highest water demand for agricultural irrigation).The arrows show the points of the aquifer where higher phreatic levels occurred during the summer. 
La escasa profundidad del nivel freático en el área de estudio (valor medio anual \pm desviación estándar: $4.3 \pm 1.9$ m; Fig. 2) y la elevada permeabilidad del suelo (mayoritariamente de tipo Fluvisol, Luvisol) y del sustrato litológico (gravas arenas y limos en fondos de valle y primeras terrazas aluviales), confieren una alta vulnerabilidad al acuífero aluvial (ya referida con anterioridad por Navas et al., 1998). Las variaciones invierno-verano del nivel freático revelan una situación singular, observándose una clara inversión en la dinámica natural de recarga del acuífero en amplias zonas del mismo (Fig. 2). El notable ascenso del nivel freático durante agosto de 2005 en los puntos PH3, Ptñ3, PJ3, PJ4, PT1, PT2 y PT3 respecto a los niveles de marzo, podría explicarse por las recargas procedentes de los retornos de riego (Arauzo et al. 2007; MartínezBastida et al. 2008), puesto que apenas se regis- traron precipitaciones durante ese periodo (aporte total por lluvia: $29 \mathrm{~mm}$ ). En los puntos en los que se practica el riego por inundación, el ascenso estival del nivel freático fue significativamente superior con relación al resto de los puntos del acuífero (test $t$ de Student: $t=3.74, p<0.01$ ). La procedencia mayoritariamente fluvial de las aguas de riego en la cuenca del Jarama, en un contexto de prácticas de riego poco optimizadas y alta permeabilidad de la zona no saturada, favoreció el trasvase artificial de agua desde la red fluvial al acuífero aluvial, alterando la dinámica hídrica natural en el sistema río-acuífero.

La composición iónica del agua en la red fluvial y en el acuífero aluvial se representa en el diagrama de Piper de la figura 3. En la cuenca domina el agua sulfatada-cálcica (con ciertas variaciones en concordancia con el contexto geológico local), si bien el río Manzanares presenta un

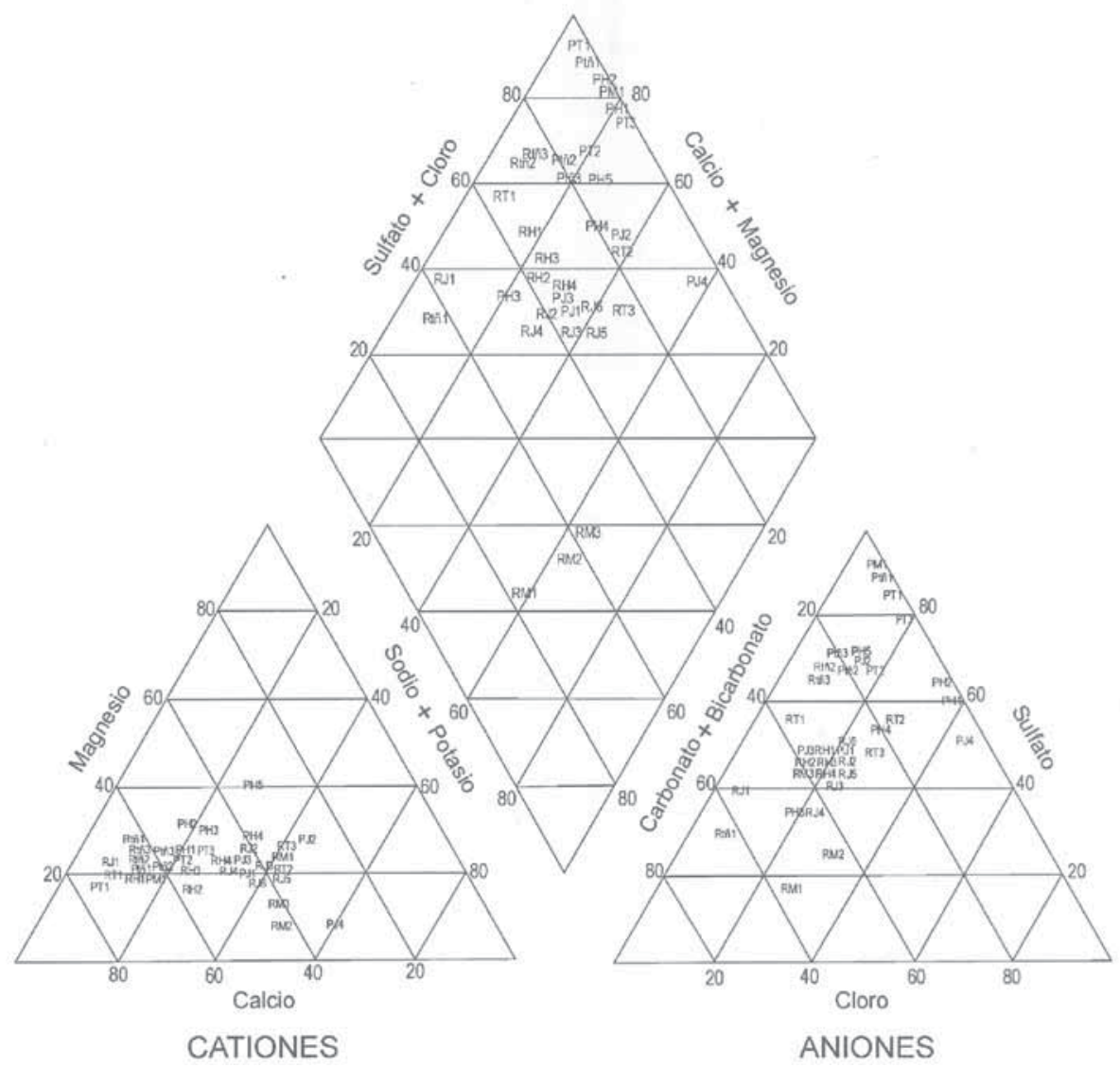

Figura 3. Diagrama de Piper. Piper diagram. 

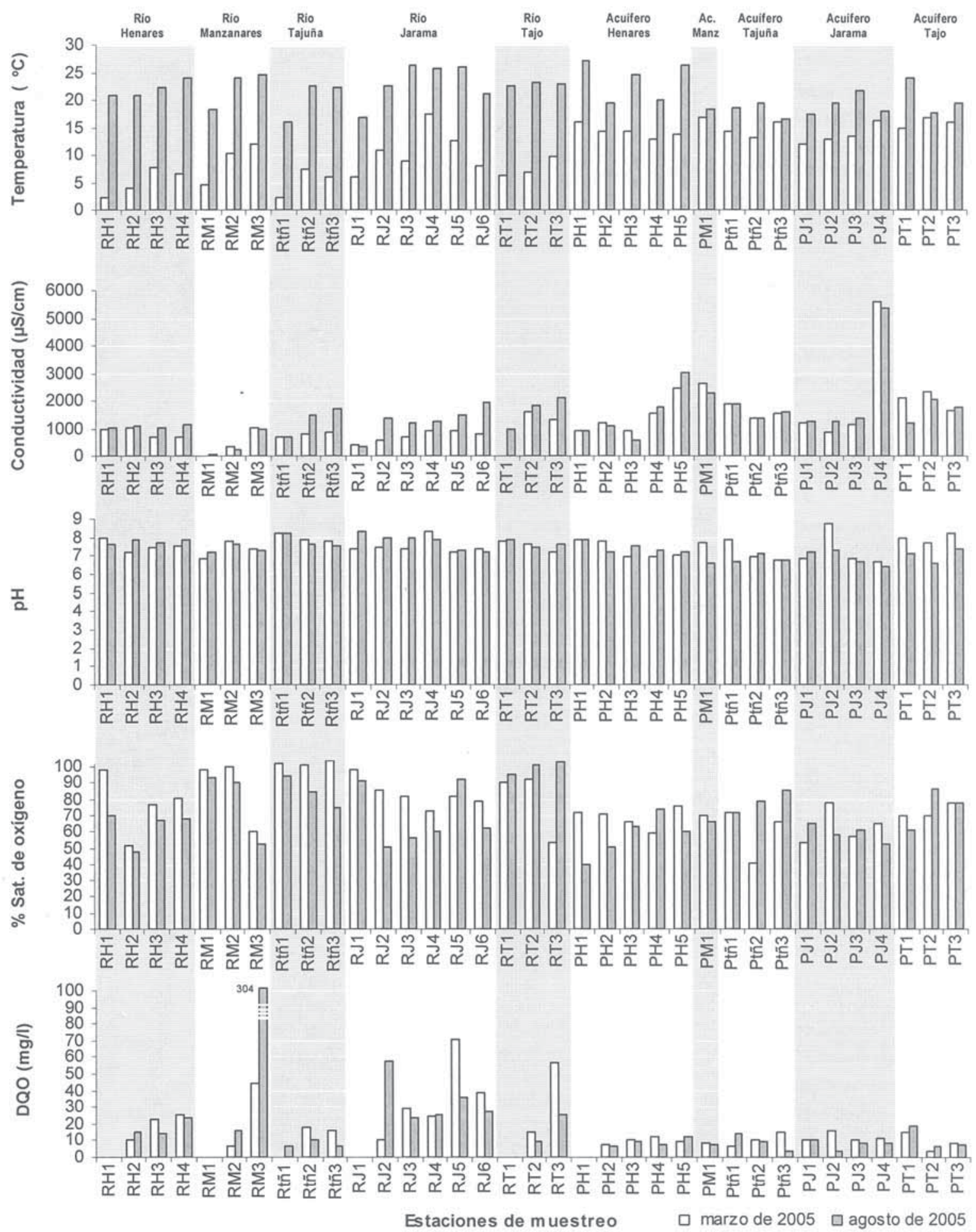

Figura 4. Temperatura del agua, conductividad eléctrica, pH, porcentaje de saturación de oxígeno y DQO en los ríos y en el acuífero aluvial al final del invierno y durante el verano de 2005. Water temperature, electric conductivity, pH, percentage of oxygen saturation and QOD in the rivers and alluvial aquifer at the end of the winter and during the summer of 2005. 
agua de tipo bicarbonatada sódica y las cabeceras del Jarama y el Tajuña de tipo bicarbonatada cálcica (Tabla 1). La clasificación según la dureza del agua muestra tipologías que varían de dura a muy dura, tanto en los ríos como en el acuífero, excluyendo las zonas de cabecera y tramo medio del río Manzanares, de tipo muy blanda a blanda (Tabla 1). En las figuras 4 y 5 se representan los parámetros de calidad analizados en la red fluvial y en el acuífero aluvial. En general, se observa un buen nivel de calidad del agua en las zonas altas del sistema río-acuífero. Sin embargo, en los puntos RM3, Rtñ2, Rtñ3, RJ3, RJ4, RJ5, RJ6, RT2 y RT3 de la red fluvial no se alcanzan los objetivos de calidad establecidos en el Plan Hidrológico de la Cuenca del Tajo (R.D. 1664/1998; Orden 18236 de 13 de agosto de 1999). Se observan valores muy elevados de amonio y DQO en el tramo bajo del río Manzanares, el tramo medio-bajo del Jarama y el tramo del Tajo posterior la incorporación del Jarama, atribuibles a la incorporación de efluentes de aguas residuales tratadas, procedentes de las principales núcleos urbanos (Fig. 1). En el tramo bajo del río Manzanares también se excede el límite fijado para el fósforo (igualmente explicable por los aportes urbanos). Los tramos medio del Henares y medio-bajo del Tajuña, localizados en el área de influencia de facies margo-yesíferas del Mioceno, presentan valores de conductividad eléctrica por encima del valor de referencia. Respecto a la calidad del agua en el acuífero aluvial, la conductividad y la concentración de nitrato también exceden los límites establecidos como objetivos de calidad en el Plan Hidrológico en una parte considerable de su extensión (Fig. 4 y 5), lo cual imposibilita su uso como reserva para abastecimiento y confiere un grado de restricción para el uso en riego agrícola de moderado a severo (Ayers \& Westcot, 1985).

En la red fluvial el nitrógeno total presentó una distribución creciente desde las zonas de cabecera hacia los tramos bajos de cada subcuenca (Fig. 5). En el acuífero aluvial, sin embargo, no se apreció siempre un patrón similar, apareciendo áreas con mayores concentraciones de nitrógeno en los tramos medios de las subcuencas del Henares y el Tajuña (Fig. 5). Las diferentes formas químicas del nitrógeno tampoco mostraron una distribución uniforme en el sistema río-acuífero. Al ser la zona no saturada un sistema oxidante abierto, la forma química dominante en todo el acuífero aluvial es el nitrato (Fig. 5). Sin embargo, en los tramos medios y/o bajos de los ríos Manzanares, Jarama, Henares y Tajo (después de la incorporación del Jarama) el amonio es con frecuencia más abundante que el nitrato y las concentraciones de nitrito son generalmente muy elevadas (Fig. 5), en concordancia con los valores máximos de DQO y fósforo total y los valores más bajos en saturación de oxígeno (Fig. 4 y 5), parámetros todos ellos que indican la incorporación de aguas residuales urbanas con un mayor o menor grado de tratamiento.

En la figura 6 se muestran los mapas de distribución del contenido en nitrato en el acuífero aluvial de la cuenca del Jarama y parte de la cuenca del Tajo (a su paso por la Comunidad de Madrid) durante las dos campañas de muestreo. Se estima que el $36 \%$ del área total del acuífero aluvial presentó concentraciones de nitrato superiores a $50 \mathrm{mg} / \mathrm{l}$ (límite máximo establecido por la Directiva 91/676/CEE), el $25 \%$ entre 25 y $50 \mathrm{mg} / \mathrm{l}$ (la Directiva 75/440/CEE establece un límite guía de $25 \mathrm{mg} / \mathrm{l})$, y únicamente en el $39 \%$ se registraron concentraciones inferiores a $25 \mathrm{mg} / \mathrm{l}$. La distribución espacial del nitrato no presentó incrementos importantes entre el final del invierno y el periodo estival, salvo en el tramo medio del Tajuña, el tramo bajo del Jarama y el tramo del Tajo posterior a la incorporación del Jarama, con concentraciones de nitrato entre 75 y $125 \mathrm{mg} / \mathrm{l}$. Arauzo et al. (2006a y b) observaron una dinámica inviernoverano en la concentración de nitrato mucho más intensa en los acuíferos aluviales de la cuenca del Oja-Tirón (La Rioja-Castilla y León) explicable, en ese caso, por la alta tasa de recarga de los mismos al final del invierno, que permitía cierta recuperación temporal.

A diferencia del acuífero aluvial, el nitrógeno en los ríos de la cuenca del Jarama aparece bajo distintas formas químicas (Fig. 5), de ahí que en la figura 6 se opte por la representación como nitrógeno total. Ya se ha mencionado el probable origen urbano del nitrógeno en los tramos contaminados de los ríos (Fig. 6), sólo cabe precisar que no se aprecian diferencias importantes en los 

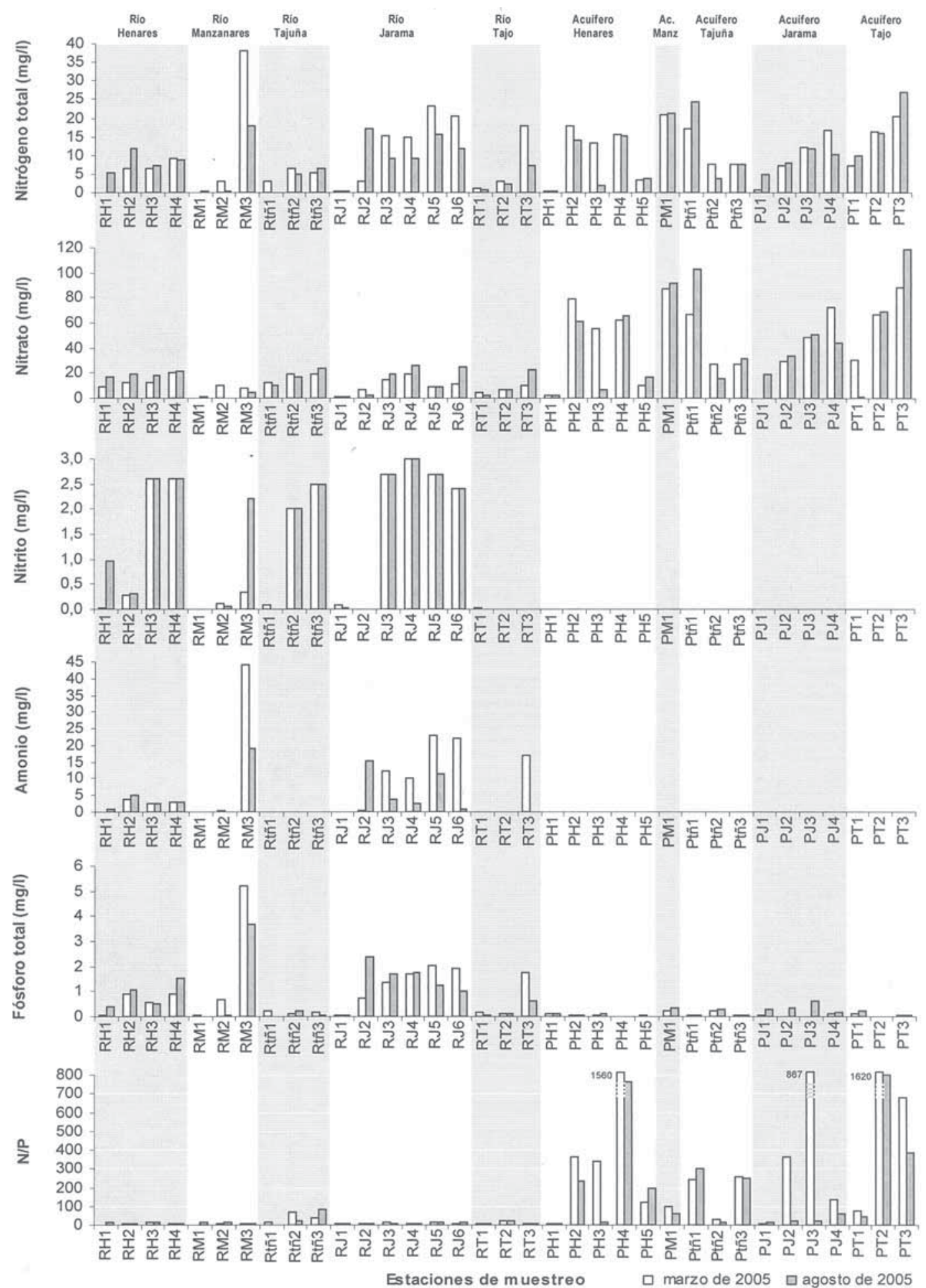

Figura 5. Nitrógeno total, nitrato, nitrito, amonio, fósforo total y cociente N:P en los ríos y en el acuífero aluvial al final del invierno y durante el verano de 2005. Total nitrogen, nitrate, nitrite, ammonia, total phosphorous and N:P ratio in the rivers and alluvial aquifer at the end of the winter and during the summer of 2005. 


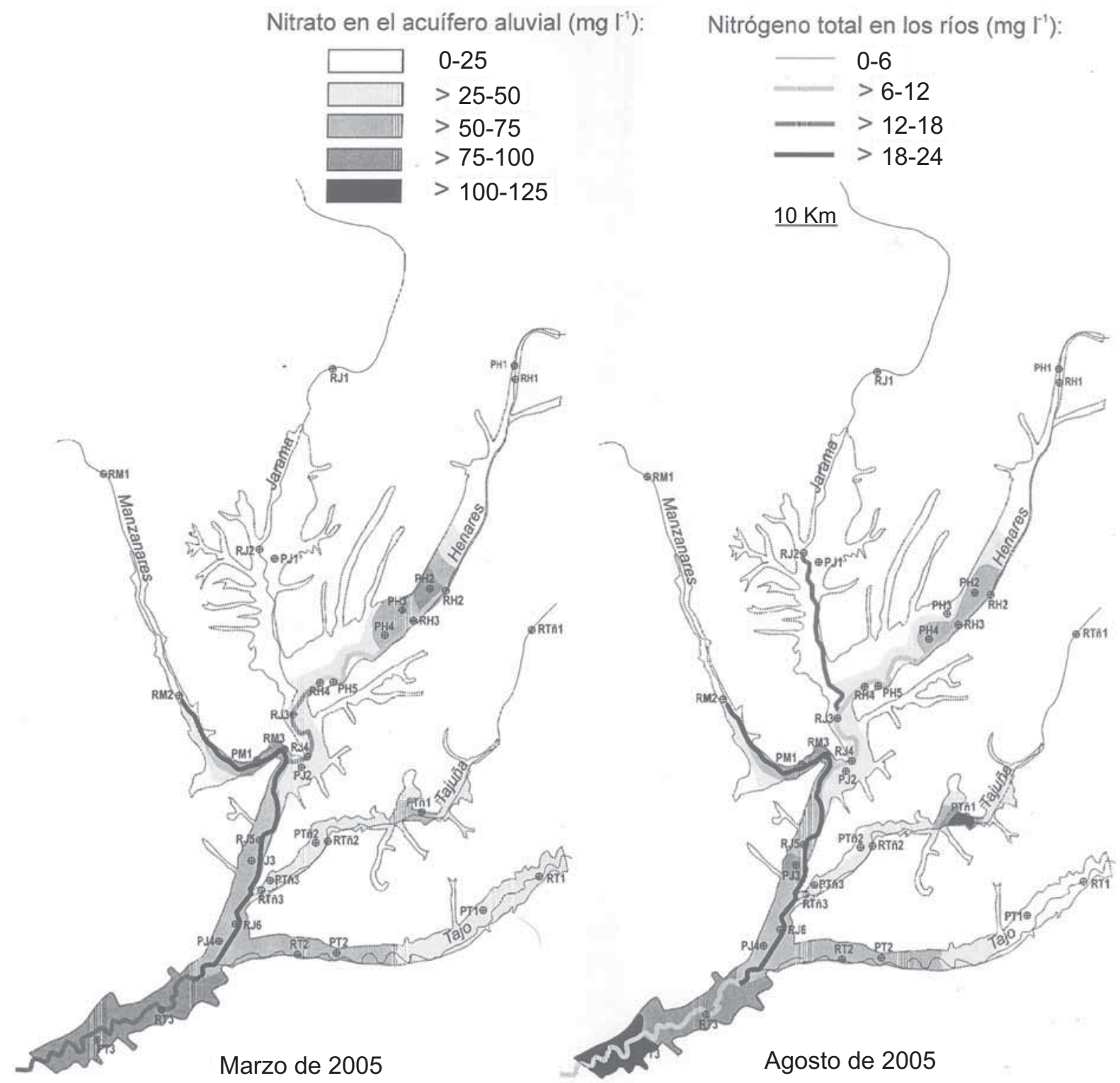

Figura 6. Distribución del contenido en nitrato en el acuífero aluvial y del contenido en nitrógeno total en los ríos, al final del invierno y durante el verano de 2005. Nitrate content distribution in the alluvial aquifer and total nitrogen content in the rivers, at the end of the winter and during the summer of 2005.

niveles de contaminación fluvial por nitrógeno entre el invierno el verano.

En áreas tan antropizadas como la que nos ocupa existe un amplio grado de incertidumbre en el diagnóstico de las fuentes de contaminación difusa de los recursos hídricos. Una primera aproximación visual nos ha permitido apreciar el solapamiento entre la distribución de las áreas destinadas al regadío en la Comunidad de Madrid (Fig. 1) y las zonas que presentan máximas concentraciones de nitrato en el acuífero aluvial (Fig. 6). Las correlaciones significativas entre la concentración de nitrato en el acuífero y la extensión dedicada al regadío aguas arriba de cada pozo, y entre la concentración de nitrato y el cociente N/P en todas las estaciones (Tabla 2) vienen 
Tabla 2. Correlaciones de Pearson entre la concentración de nitrato en las estaciones de muestreo del acuífero y la superficie de regadío aguas arriba de cada pozo, y entre la concentración de nitrato y el cociente N/P en todas las estaciones de muestreo, durante las campañas de marzo y agosto de 2005. *: Significación estadística para $p<0.05$; ***: Significación estadística para $p<0.001$. Pearson correlations between the nitrate concentration in the sampling stations of the aquifer and the upstream irrigation area for each well, and between the nitrate concentration and the N/P ratio in all sampling stations, during March and August 2005 campaigns. *: Statistical significance for $\mathrm{p}<0.05$; ***: Statistical significance for $\mathrm{p}<0.001$.

\begin{tabular}{lcc}
\hline & $\begin{array}{c}{\left[\mathrm{NO}_{3}^{-}\right]} \\
\text {marzo 2005 }\end{array}$ & $\begin{array}{c}{\left[\mathrm{NO}_{3}^{-}\right]} \\
\text {agosto 2005 }\end{array}$ \\
\hline $\begin{array}{l}\text { Superficie de regadío } \\
\text { aguas arriba de cada pozo } \\
\text { (estaciones de acuífero) }\end{array}$ & $0.61^{*}(n=16)$ & $0.49^{*}(n=16)$ \\
$\begin{array}{l}\text { N/P (estaciones fluviales y } \\
\text { de acuífero) }\end{array}$ & $0.59^{* * *}(n=35)$ & $0.60^{* * *}(n=27)$ \\
\hline
\end{tabular}

a reforzar esta hipótesis. El cociente N/P se ha utilizado en este caso como un indicador del origen agrícola o urbano del nitrógeno (véase Metodología), prescindiendo de su uso común como indicador del factor limitante para el desarrollo de procesos de eutrofización. En la mayoría de los suelos españoles el fósforo se encuentra en forma de fosfatos tricálcicos, insolubles en agua, que lentamente pasan a la solución del suelo. La escasa solubilidad del fósforo en este tipo de suelos confiere al cociente N/P un interesante valor como indicador de la existencia de procesos de lixiviación de nitrato procedente de los fertilizantes hacia las masas de agua de la zona saturada. En la figura 5 se observan valores bajos en el cociente N/P en los ríos y en los tramos altos del acuífero, correspondientes a zonas no contaminadas o a zonas contaminadas por nitrógeno de procedencia urbana. El acuífero aluvial, sin embargo, presenta valores muy elevados en los tramos medios y/o bajos de cada una de las subcuencas (Fig. 5), en concordancia con las áreas destinadas al regadío.

En términos generales puede decirse que la contaminación por nitrato del acuífero aluvial es atribuible a la lixiviación de los fertilizantes, debido a las malas prácticas de riego y abonado, al sustrato muy permeable y a la escasa profundidad del nivel freático. Debe excluirse la zona del acuífero en el tramo bajo del Manzanares, con escasa dedicación a la agricultura, niveles muy elevados de contaminación fluvial y cociente N/P moderado, datos que apuntan más bien a un origen urbano del nitrógeno. En el tramo mediobajo del Jarama y el tramo del Tajo posterior a la incorporación del Jarama debe considerarse que el nitrógeno del acuífero aluvial procede de una fuente difusa de tipo mixto (efecto aditivo agrícola y urbano; Fig. 6). En estos tramos el riego en exceso con agua fluvial contaminada por nitrógeno de origen urbano, genera retornos de riego en los que se suma la carga de nitrógeno lixiviado procedente de los fertilizantes y la del agua de riego (Esteller, 2002).

A pesar de la complejidad del área de estudio debido a la intensa presión antrópica, estos resultados proporcionan una base científica preliminar para considerar la posible declaración de los aluviales cuaternarios de la cuenca del Jarama como Zona Vulnerable a la contaminación por nitrato de origen agrícola en la Comunidad de Madrid, tal como indica la Directiva 91/676/CEE. La Directiva Marco del Agua no sólo establece como uno de sus objetivos el conocimiento de los procesos de interacción entre las aguas superficiales y subterráneas a escala de cuenca, sino también el estudio de los flujos de los contaminantes entre las distintas partes del ciclo hidrológico y del impacto sobre los ecosistemas acuáticos y terrestres. Iniciar la aplicación de "buenas prácticas agrícolas" en las Vegas de la Comunidad de Madrid y generalizar la depuración terciaria en las áreas urbanas de la cuenca, son iniciativas esenciales para el cumplimiento la normativa europea respecto a la concentración de nitrógeno en las aguas del sistema "río-acuífero aluvial" de la cuenca del Jarama.

\section{CONCLUSIONES}

1. Se ha realizado una caracterización hidroquímica del sistema "río-acuífero aluvial" situado en los depósitos cuaternarios de la cuenca del Jarama (Comunidad de Madrid, España), incluyendo el río Jarama, sus afluentes Henares, Manzanares y Tajuña, una parte del río Tajo (a su paso por la Comunidad de Madrid), y el acuífero alu- 
vial asociado a dicha red fluvial. Se han determinado los niveles de contaminación por nitrógeno, se han identificado las formas químicas de nitrógeno dominantes en cada parte del sistema río-acuífero y se ha evaluado el papel de los usos agrícolas y urbanos como fuente de nitrógeno en el proceso de contaminación del agua.

2. La naturaleza permeable de los sustratos aluviales, la escasa profundidad del nivel freático y las labores agrícolas de fertilización y riego en exceso, son los factores principales que determinan el desarrollo de los procesos lixiviación de nitrato. En el área de estudio confluyen estos factores de riesgo, lo cual confiere un alto grado de vulnerabilidad para sus recursos hídricos.

3. La procedencia mayoritariamente fluvial de las aguas de riego favoreció el trasvase de agua desde la red fluvial al acuífero aluvial, alterándose la dinámica hídrica natural del sistema río-acuífero. Las variaciones invierno-verano del nivel freático revelaron una inversión en la dinámica natural de recarga del acuífero, observándose un ascenso de nivel en algunas zonas durante el verano relacionado con el riego.

4. En la red fluvial el nitrógeno total presentó una distribución creciente desde las zonas de cabecera hacia los tramos bajos de cada subcuenca. En el acuífero aluvial no se apreció un patrón similar, existiendo una clara concordancia con la distribución de las zonas dedicadas a regadío.

5. La forma química de nitrógeno dominante en el acuífero aluvial fue el nitrato (de origen agrícola). En los tramos medios y/o bajos de los ríos Manzanares, Jarama, Henares y Tajo (después de la incorporación del Jarama) el amonio fue con frecuencia más abundante que el nitrato, y el nitrito presentó concentraciones elevadas (de origen urbano).

6. A partir de los mapas de distribución del contenido en nitrato en el acuífero aluvial se estima que el $36 \%$ del mismo presentó concentraciones superiores a $50 \mathrm{mg} / \mathrm{l}$, el $25 \%$ entre 25 y $50 \mathrm{mg} / \mathrm{l}$, y el $39 \%$ inferiores a $25 \mathrm{mg} / \mathrm{l}$.
7. El cociente $\mathrm{N} / \mathrm{P}$, utilizado como indicador del origen agrícola o urbano del nitrógeno, presentó valores bajos en los ríos y en los tramos altos del acuífero (zonas no contaminadas, o contaminadas por nitrógeno de procedencia urbana). En el acuífero aluvial se registraron valores muy elevados en los tramos medios y/o bajos de cada una de las subcuencas, en concordancia con las áreas destinadas a regadío. El origen mixto (agrícola y urbano) del nitrógeno en el acuífero, en tramo medio-bajo del Jarama y en la zona del Tajo posterior a la incorporación del Jarama, se explica por el efecto aditivo de la carga de nitrógeno lixiviado de los fertilizantes y del nitrógeno del agua de riego (procedente de tramos fluviales contaminados por efluentes urbanos).

8. Los resultados de este trabajo proporcionan una base científica para considerar la posible declaración de los aluviales cuaternarios de la cuenca del Jarama como Zona Vulnerable a la contaminación por nitrato de origen agrícola en la Comunidad de Madrid, tal como se establece en la Directiva 91/676/CEE.

\section{AGRADECIMIENTOS}

Este Proyecto de Investigación ha sido financiado por la Consejería de Educación de la Comunidad de Madrid, el Fondo Europeo para Desarrollo Regional y el Fondo Social Europeo (Ref.: GR/AMB/0745/2004). La Consejería de Educación de la Comunidad de Madrid y el Fondo Social Europeo han colaborado con la dotación de una beca de Formación de Personal Investigador.

\section{BIBLIOGRAFÍA}

ABRANTES, N., R. PEREIRA \& F. GONCALVES. 2006. First step for an ecological risk assessment to evaluate the impact of diffuse pollution in lake Vela (Portugal). Environ. Monitoring Assessment, 117: 411-431.

APHA 1998. Standard methods for the examination of water and wastewater. $20^{\text {th }} \mathrm{Ed}$. American Public 
Health Association, American Water Works Association and Water Environment Federation. USA. $1134 \mathrm{pp}$.

ARAUZO, M., J. J. MARTÍNEZ-BASTIDA y M. VALLADOLID. 2006a. Dinámica espacio-temporal del contenido en nitrato de las aguas superficiales y subterráneas en la cuenca del río Oja (La Rioja, España): vulnerabilidad del acuífero aluvial. Limnetica, 25: 753-762.

ARAUZO, M., M. VALLADOLID y J. J. MARTÍNEZ-BASTIDA. 2006b. Contaminación por nitrato en los acuíferos aluviales del Oja y el Tirón. Una aproximación a escala de cuenca. CCMACSIC. Madrid. 59 pp.

ARAUZO, M., J. A. DÍEZ, J. J. MARTÍNEZ-BASTIDA, M. VALLADOLID y P. HERNÁIZ. 2007. Comparación de un método directo y un método indirecto para la estimación del drenaje y el balance hídrico en la zona no saturada. En: Estudios de la zona no saturada, Vol. VIII, ZNS'07. J. V. Giráldez y F. J. Jiménez (eds.): 77-82. Universidad de Córdoba, CSIC, Junta de Andalucía. Córdoba.

AYERS, R. S. \& D. W. WESTCOT. 1985. Water quality for agriculture. FAO Irrigation and Drainage. Paper, n. ${ }^{\circ}$ 29. Food and Agriculture Organization of the United Nations. Rome. 174 pp.

BALL, D. F., A. M. MACDONALD \& A. LILLY. 2005. Agriculture and diffuse pollution: groundwater nitrate vulnerable zones in Scotland. Scottish J. Geology, 41: 61-68.

COLLEGE OF AGRICULTURAL SCIENCES, AGRICULTURAL RESEARCH AND COOPERATIVE EXTENSION. 2001. Managing phosphorus for agriculture and the environment. The Pennsylvania State University, USA. 16 pp.

CONFEDERACIÓN HIDROGRÁFICA DEL TAJO. 2005a. Redes de control. Calidad de agua. www.chtajo.es/redes/calidad.htm.

CONFEDERACIÓN HIDROGRÁFICA DEL TAJO. 2005b. Informe anual de las aguas subterráneas de la Confederación Hidrográfica del Tajo, año 2005. Confederación Hidrográfica del Tajo, Madrid. Memoria de 23 pp. +10 Anexos.

ESTELLER, M. V. 2002. Vulnerabilidad de acuíferos frente al uso de aguas residuales y lodos en agricultura. Revista Latino-Americana de Hidrogeología, 2: 103-113.

NEAL, C. \& H. P. JARVIE. 2005. Agriculture, community, river eutrophication and the water framework directive. Hydrol. Processes, 19: 1895-1901.
FAO/CEPE. 1991. Legislation and Measures for the Solving of Environmental Problems Resulting from Agricultural Practices (With Particular Reference to Soil, Air and Water), Their Economic Consequences and Impact on Agrarian Structures and Farm Rationalization. Agri/Agrarian Structures and Farm Rationalization, Report $N^{\circ} 7$. United Nations publication, Geneve. 53 pp.

FORMAN, D. 2004. Commentary: Nitrites, nitrates and nitrosation as causes of brain cancer in children: epidemiological challenges. Internat. J. Epidemiology, 33: 1216-1218.

GERRA, A. y F. MONTURIOL. 1970. Mapa de suelos de la provincia de Guadalajara. Escala 1:250.000. Instituto Nacional de Edafología y Agrobiología José María Albareda, CSIC, Madrid. Memoria de 89 pp. + 1 mapa.

HEATHWAITE, A. L., P. F. QUINN \& C. J. M. HEWETT. 2005. Modelling and managing critical source areas of diffuse pollution from agricultural land using flow connectivity simulation. J. Hydrol., 304: 446-461.

HALL, M. D., M. J. SHAFFER, R. M. WASKOM \& J. A.DELGADO. 2001. Regional nitrate leaching variability: What makes a difference in northeastern Colorado. J. Am. Water Res. Ass., 37: 130-150.

ITGME-CAM 1988. Atlas geocientífico del medio natural de la Comunidad de Madrid. Ed: Instituto Tecnológico Geominero de España y Comunidad de Madrid. Madrid. Memoria de 83 pp. +7 mapas.

INSTITUTO TECNOLÓGICO GEOMINERO DE ESPAÑA. 1998. Mapa de contenido en nitrato de las aguas subterráneas en España. Escala 1:1.500.000. Ministerio de Medio Ambiente. Madrid. Memoria de 45 pp. + 1 mapa.

KNAPP, M. F. 2005. Diffuse pollution threats to groundwater: a UK water company perspective. Quarterly J. Engin. Geol. Hydrogeol., 38: 39-51.

LANGE. 1998. Handbook of photometrical operation analysis. LASA/IP/CADAS/ISIS. Germany. 320 pp.

MAPYA 2005. Mapa de cultivos y aprovechamientos de España. Escala 1:50.000. Ministerio de Agricultura, Pesca y Alimentación. www.mapa.es

MARTÍNEZ-BASTIDA, J. J., M. ARAUZO, J. A. DÍEZ, M. VALLADOLID y P. HERNÁIZ. 2008. The influence of irrigation on soil water dynamics and nitrate leaching. En: 15th $N$ Workshop: Towards a better efficiency in $N$ use: (en prensa). Universidad de Lleida, RUENA. Lleida. 
MINISTERIO DE MEDIO AMBIENTE. 2001. Caracterización de las fuentes agrarias de contaminación de las aguas por nitratos. Secretaría de estado de Aguas y Costas, Dirección General de Obras Hidráulicas y Calidad de Aguas. Madrid. Memoria de 151 pp. + 11 mapas.

MONTURIOL, F. y L. ALCALÁ. 1990. Mapa de capacidad potencial de uso agrícola de la Comunidad de Madrid. Escala 1:200.000. Consejería de Agricultura y Cooperación, Comunidad de Madrid y Consejo Superior de Investigaciones Científicas. Madrid. Memoria de 3 pp. + 1 mapa.

MORALES-SUÁREZ, M. M., A. LLOPIS-GONZÁLEZ \& M. L. TEJERIZO-PÉREZ.] 1995. Impact of nitrates in drinking-water on cancer mortality in Valencia, Spain. Europ. J. Epidemiol., 11: 15-21.

MURPHY, J. \& J. P. RILEY. 1962. A modified single solution method for the determination of phosphate in natural waters. Anal. Chim. Acta, 27: 31-36.

NAVAS, E., M. GARCÍA, R. LLAMAS y J. VRBA. 1998. La vulnerabilidad de las aguas subterráneas en el Plan Regional de la Comunidad Autónoma de Madrid. En: Jornadas sobre la contaminación de las aguas subterráneas: un problema pendiente: 429-436. AIH-GE. Valencia.
NIXON, S. C., T. J. LACK, D. T. E. HUNT, C. LALLANA \& A. F. BOSCHET. 2000. ¿Es sostenible el uso del agua? Situación, perspectivas y problemas. Informe de evaluación ambiental, Agencia Europea de Medio Ambiente. Copenhague. 36 pp.

SAN DIEGO-MCGLONE, M. L., V. S. SMITH \& V. F. NICOLAS. 2000. Stoichiometric interpretations of $\mathrm{C}: \mathrm{N}: \mathrm{P}$ ratios in organic waste material. Marine Poll. Bulletin, 40: 325-330.

SANDOR, J., I. KISS, O. FARKAS \& I. EMBER. 2001. Association between gastric cancer mortality and nitrate content of drinking water: Ecological study on small area inequalities. Europ. J. Epidemiol., 17: 443-447.

THORPE, N. \& A. SHIRMOHAMMADI A. 2005. Herbicides and nitrates in groundwater of Maryland and childhood cancers: A geographic information systems approach. J. Environ. Sci. and Health Part C-Environmental Carcinogenesis \& Ecotoxicology Reviews, 23: 261-278.

VARELA, M. 1994. Nitrate contamination of drinking water source. International Workshop of health aspects of nitrate and its metabolite (particularly nitrite). Council of Europe. RIVM. Bilthoven, 8-10 noviembre 1994. 